\title{
Sub-Chandrasekhar models for Type la supernovae and astrophysical transients
}

\section{Fink ${ }^{*}{ }^{a}$ F. K. Röpke, ${ }^{a}$ S. A. Sim, ${ }^{b}$ M. Kromer, ${ }^{c}$ I. R. Seitenzahl, ${ }^{a}$ A. J. Ruiter ${ }^{c}$ and W. Hillebrandt ${ }^{c}$}

${ }^{a}$ Institut für Theoretische Physik und Astrophysik, Universität Würzburg, Emil-Fischer-Straße 31, 97074 Würzburg, Germany

${ }^{b}$ Research School of Astronomy and Astrophysics, Mount Stromlo Observatory, Cotter Road, Weston Creek, ACT 2611, Australia

${ }^{c}$ Max-Planck-Institut für Astrophysik, Karl-Schwarzschild-Straße 1, 85748 Garching, Germany

E-mail: mfinkeastro.uni-wuerzburg.de,

friedrich.roepkedastro.uni-wuerzburg.de, ssimemso.anu.edu.au, mkromer@mpa-garching.mpg.de, iseitenzahleastro.uni-wuerzburg.de, a jr@mpa-garching.mpg.de, wfh@mpa-garching.mpg.de

\begin{abstract}
Sub-Chandrasekhar models aim to explain Type Ia supernovae from the explosion of white dwarf stars with masses below the Chandrasekhar limit. An advantage of this scenario is the wide range of possible progenitor masses, which may lead to a wide range of explosion brightnesses. We study sub-Chandrasekhar models by means of hydrodynamic simulations, nucleosynthetic post-processing and multidimensional radiative transfer calculations. We find that an assumed detonation in an accreted He layer likely triggers a secondary detonation in the carbon-oxygenwhite dwarf core, which leads to a complete explosion of the white dwarf star. In models with normal brightnesses $\left(M_{\text {core }} \gtrsim 0.8 M_{\odot}\right)$, the He detonation nucleosynthesis is, however, problematic for the observable predictions. We discuss potential changes to the model that may lead to an improvement in this respect. As a second scenario, we study explosions in systems with lowmass carbon-oxygen cores as candidates for faint astrophysical transients. For this scenario, the He detonation nucleosynthesis may be compatible with the observations. The occurrence of a secondary core detonation is shown to potentially leave an observable imprint in the light curves. Finally, we also discuss our models in the context of Galactic chemical evolution.
\end{abstract}

XII International Symposium on Nuclei in the Cosmos

August 5-12, 2012

Cairns, Australia

${ }^{*}$ Speaker. 


\section{Introduction}

There is common agreement that Type Ia supernovae (SNe Ia) are thermonuclear explosions of carbon-oxygen-white dwarf (CO-WD) stars that accrete matter from a binary companion. The exact nature of the progenitor systems and the explosion mechanism are, however, still unknown. $\mathrm{SNe}$ Ia are important for astrophysics for a variety of reasons. They are successfully applied as standardisable candles for cosmological distance measurements. As the explosion produces and ejects a significant amount of iron-group elements (IGEs), SNe Ia also have a strong impact on Galactic chemical evolution. It is important to recognise that $\mathrm{SNe}$ Ia do not form a perfectly homogeneous class of events. Apart from approximately $70 \%$ of so-called normal events [1], which are fairly homogeneous e.g. in their spectral properties, there are also many peculiar subtypes. Nowadays, automated surveys also find a growing number of faint transients, which are also believed to be of thermonuclear origin. One of the main goals of current studies is to explain the whole variety of observations, which likely requires more than one progenitor channel. The sub-Chandrasekhar model is especially interesting in this context, as it can cover a wide range of brightnesses from very luminous $\mathrm{SNe}$ Ia down to faint transients.

\section{Sub-Chandrasekhar models as candidates for normal Type Ia supernovae}

In the sub-Chandrasekhar scenario, He-rich matter accumulates in a shell around a sub-Chandrasekhar (most likely CO) WD core due to accretion at relatively low rates. By the liberation of gravitational energy, the degenerate matter in this shell is heated and, if the accretion rate is low enough, a significant amount of He-rich matter $M_{\text {sh,ign }}$ accumulates before a shell flash ensues. If $M_{\text {sh,ign }}$ is large enough, the shell flash may evolve as violent detonation. Such a shell detonation can potentially trigger a secondary detonation in the WD core either

(a) directly, when the He-detonation shock hits the edge of the core (so-called edge-lit doubledetonation scenario or $E L D D)$, or

(b) delayed, after shocks sent from the He detonation into the core from all directions converge somewhere off-centre (converging-shock double-detonation scenario or CSDD).

In both cases, a full explosion of the star ensues with a brightness that is mainly determined by the total mass of the progenitor. These so-called double-detonation sub-Chandrasekhar models are potential candidates for normal SNe Ia and discussed below. In the alternative scenario of a pure He shell detonation that fails to trigger a core detonation, only relatively faint events could be the outcome (He-only detonation or $\mathrm{HeD}$; also known as point-Ia scenario [2, 3], as at maximum, these explosions reach roughly one tenth of the brightness of a SN Ia).

\subsection{Advantages of the model}

One of the main advantages of sub-Chandrasekhar models is the frequency of their potential progenitor systems. In this scenario, significantly less mass has to be accreted onto the primary WD than in the standard Chandrasekhar mass $\left(\mathrm{M}_{\mathrm{Ch}}\right)$ models. Thus, current population synthesis calculations predict that sub-Chandrasekhar mass double-detonations (in contrast to $\mathrm{M}_{\mathrm{Ch}}$ models) may explain a significant fraction of the SN Ia rate [4]. 
A prerequisite for this prediction is the robustness of the explosion mechanism. For an assumed He shell detonation, it has been shown that the delayed core detonation (CSDD) is achieved very robustly — even in the case of minimum He detonation strength [5] (of course, in some cases an ELDD might already trigger the core detonation). For models with CO-WD core masses above $0.8 \mathrm{M}_{\odot}$, the same study has found a wide range of ${ }^{56} \mathrm{Ni}$ masses from $0.17 \mathrm{M}_{\odot}$ up to $1.1 \mathrm{M}_{\odot}$. As the secondary core detonation seems to work independent of the core mass, the mass of the exploding star could be the system parameter that may explain the range of observed SN Ia brightnesses or even the correlations of SN Ia brightnesses with their host stellar populations [6].

\subsection{Shortcomings of current models}

Most shortcomings of sub-Chandrasekhar models are associated with the nucleosynthesis products of the detonation in the He shell. Due to the lower nuclear binding energy of $\mathrm{He}$ (compared with CO), IGEs are still produced at the low densities in the outer shell. ${ }^{56} \mathrm{Ni}$ in the outer ejecta layers has been shown to be in conflict with observed early spectra of SNe Ia [7, 8]. However, in previous studies $[9,10]$, relatively thick shells with masses $M_{\text {sh,ign }} \gtrsim 0.2 \mathrm{M}_{\odot}$ and with high densities $\rho_{\mathrm{b}} \gtrsim 10^{6} \mathrm{~g} \mathrm{~cm}^{-3}$ at the base were studied. In the above mentioned more recent study [5], minimum shell masses that might still detonate (as determined by Bildsten et al. [2]) were investigated. These models had significantly lower shell densities $\left(3.7 \times 10^{5} \mathrm{~g} \mathrm{~cm}^{-3} \leq \rho_{\mathrm{b}} \leq 8.7 \times 10^{5} \mathrm{~g} \mathrm{~cm}^{-3}\right)$ than in previous studies, resulting in $48-66 \%$ of unburnt $\mathrm{He}$ and very low ${ }^{56} \mathrm{Ni}$ masses (less than $0.0044 \mathrm{M}_{\odot}$ ) in the shell detonation products. In several of the models the shell ejecta were even dominated by other IGEs like ${ }^{52} \mathrm{Fe},{ }^{48} \mathrm{Cr}$ and ${ }^{44} \mathrm{Ti}$. But, as detailed radiative transfer simulations have shown [11], the presence of the elements $\mathrm{Cr}$ and $\mathrm{Ti}$ is also in conflict with the observations: these elements are very effective in absorbing flux at blue wavelengths of the spectrum and in reemitting flux at red wavelengths. Therefore, for all the sub-Chandrasekhar models of that study, the $B-V$ colours are too red and the early and maximum spectra are perturbed by the emission of $\mathrm{Cr}$ and $\mathrm{Ti}$, which obscure the absorption lines of intermediate-mass elements (IMEs) characteristic for normal SNe Ia [11].

\subsection{Improving the agreement}

To improve the agreement of synthetic observables with observations, the amount of IGEs in the He shell ejecta of current models has to be reduced. In general, this requires less complete burning in the He shell detonation. In the following, we discuss how this could be accomplished.

(a) Changing the initial shell composition: By accretion from the donor star, non-explosive shell burning prior to shell detonation or dredge-up processes from the underlying core, the He shell might be significantly enriched with carbon or heavier nuclei. The accretion of carbon-enriched helium material might be expected in particular if the donor star is a hybrid WD (enriched with CO) rather than pure helium. If, due to this enrichment, the ratio of $\alpha$ particles to seed particles for $\alpha$-captures drops sufficiently, the maximum mass numbers that can be achieved in the detonation is reduced. The addition of 33\% carbon has been shown to stop the $\alpha$-chain roughly at ${ }^{36} \mathrm{Ar}$ and to significantly improve the agreement of synthetic and observed spectra [11]. 


\begin{tabular}{cccccccc}
\hline Model & $\begin{array}{c}T_{\mathrm{c}} \\
(\mathrm{K})\end{array}$ & $\begin{array}{c}\rho_{\mathrm{c}} \\
\left(\mathrm{g} \mathrm{cm}^{-3}\right)\end{array}$ & $\begin{array}{c}T_{\mathrm{b}} \\
(\mathrm{K})\end{array}$ & $\begin{array}{c}\rho_{\mathrm{b}} \\
\left(\mathrm{g} \mathrm{cm}^{-3}\right)\end{array}$ & $\begin{array}{c}M_{\mathrm{CO}} \\
\left(\mathrm{M}_{\odot}\right)\end{array}$ & $\begin{array}{c}M_{\mathrm{He}} \\
\left(\mathrm{M}_{\odot}\right)\end{array}$ & $\begin{array}{c}M_{\mathrm{tot}} \\
\left(\mathrm{M}_{\odot}\right)\end{array}$ \\
\hline $\mathrm{S}$ & $5.0 \times 10^{5}$ & $8.5 \times 10^{6}$ & $5.0 \times 10^{5}$ & $1.3 \times 10^{6}$ & 0.58 & 0.21 & 0.79 \\
$\mathrm{~L}$ & $1.0 \times 10^{7}$ & $3.8 \times 10^{6}$ & $2.0 \times 10^{8}$ & $0.59 \times 10^{6}$ & 0.45 & 0.21 & 0.66 \\
\hline
\end{tabular}

Table 1: Initial model parameters. Indices $c$ and $b$ refer to the WD centre and the base of the He shell, respectively.

(b) Lowering the shell densities: Alternative accretion scenarios could allow for the initiation of a He detonation in a lower density shell, where it would produce primarily IMEs. A potential realisation of this scenario could be a double-degenerate system with dynamically unstable accretion in which the donor is a He-WD (or He-CO hybrid WD) [12]. Another possibility are violent CO-CO WD mergers (cf. e.g. [13]) in which the primary WD has a sufficiently thick remaining He shell that may be ignited during the merger.

(c) More realistic detonation modelling: In our current models, the dependence of the detonation speed on the curvature of the front [14] is not taken into account. Refining our models in this respect would result in somewhat lower velocities and less complete burning in the $\mathrm{He}$ detonation.

\section{Low-mass sub-Chandrasekhar models as candidates for faint transients}

Even without the suggested changes of Sect. 2.3, the double detonation sub-Chandrasekhar models of Fink et al. [5] are interesting candidates for faint transients, if the range of WD core masses is extended to values below $0.8 \mathrm{M}_{\odot}$. Among the observations of faint transients there are indeed indications of $\mathrm{Ca}$ and $\mathrm{Ti}$ in the spectra $[15,16]$. In the following, we summarise the results of a current study that investigates low-mass sub-Chandrasekhar models [17]. We focus on the impact of the different core detonation scenarios (see Sect. 2) on the observable outcomes.

\subsection{Hydrodynamic simulations}

We carry out hydrodynamic simulations for two different progenitor models ( $\mathrm{S}$ and $\mathrm{L}$, for standard and low-mass) with total masses of 0.79 and $0.66 \mathrm{M}_{\odot}$ (for parameters, see Table 1). For each progenitor model, we study the three explosion scenarios explained in Sect. 2: CSDD, ELDD and $\mathrm{HeD}$.

The simulations are carried out similar to Fink et al. [5]: we apply the finite volume hydrodynamics code LEAFS $[18,19]$ on $2 \mathrm{D}$ grids with $1024 \times 2048$ cells. The grid co-expands with the explosion after the onset of the $\mathrm{CO}$ detonation. Detonation burning is modelled with a level-set approach, however, the He detonation tables were newly calibrated using an improved scheme for the detonation speed and a new version of the reaction rate libraries (for details, see [17]). Detailed abundances are calculated in a post-processing step that evaluates the trajectories of approximately 65000 variable-mass tracer particles [20]. Finally, synthetic observables are obtained from 3D Monte-Carlo radiative transfer simulations with the ARTIS code [21, 22].

Based on approximate detonation criteria [23], we find that a secondary core detonation due to converging shocks (CSDD) is still likely—even for our low-mass CO cores. However, for com- 


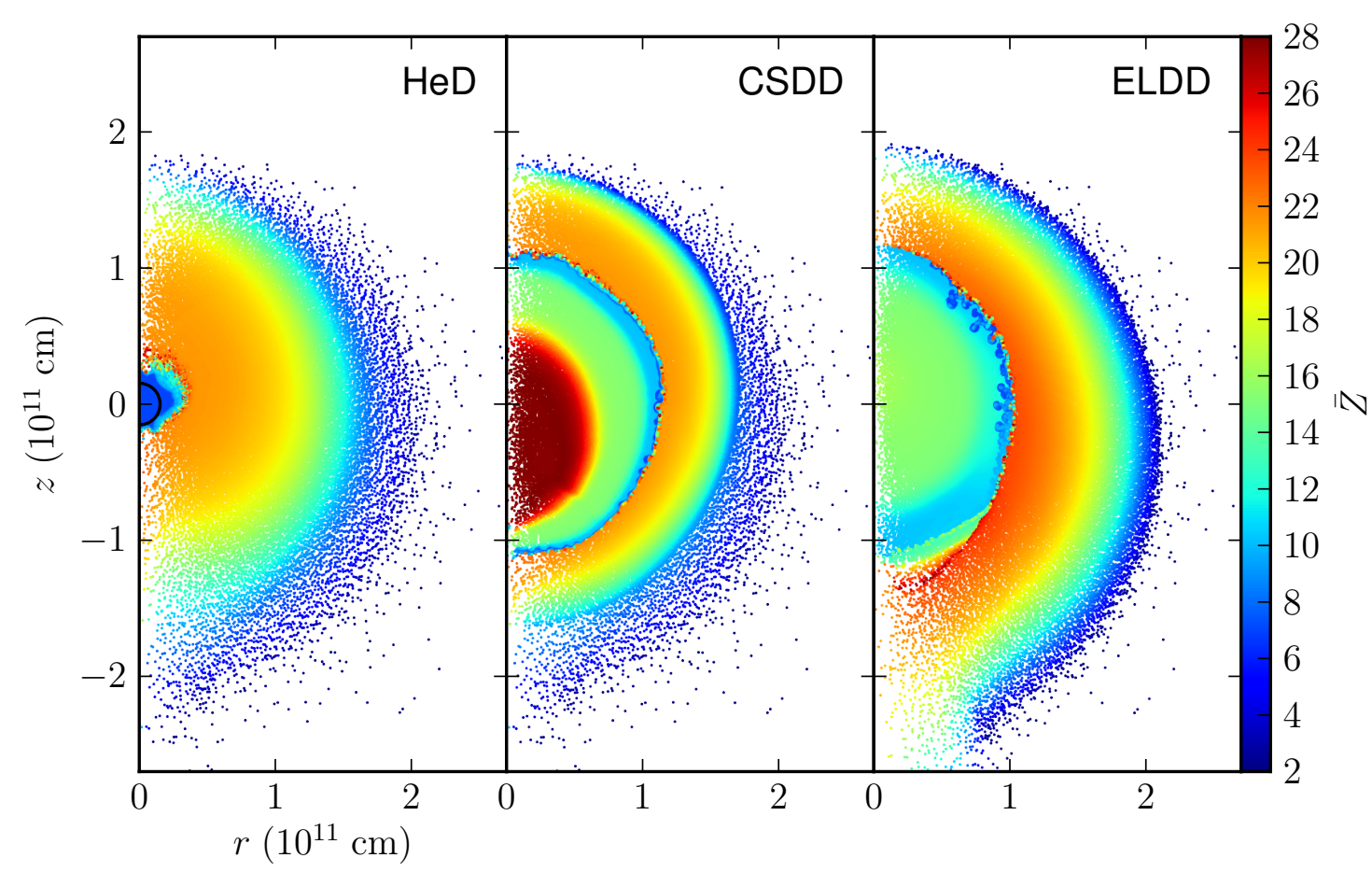

Figure 1: Final ejecta structures $(t=100 \mathrm{~s})$ for all $\mathrm{S}$ models. Shown are the mean atomic numbers of the tracer particles. A black contour indicates the bound WD core in the HeD model.

parison with published point-Ia models, we also compute a pure He detonation (HeD) model in which we inhibit the core detonation. As a third possibility, an edge-lit core detonation (ELDD) is assumed, although we find that it is only marginally possible for our setups. The final ejecta structures of all three explosion scenarios for the $\mathrm{S}$ progenitor model are shown in Fig. 1. While the HeD model leaves a WD remnant, the core detonation models completely unbind the system and thereby also accelerate the He detonation products to significantly higher velocities. Due to the pre-compression by the converging shock wave, a significant amount of ${ }^{56} \mathrm{Ni}$ is produced in the core detonation of the CSDD-S model. The ELDD-S model, which lacks this pre-compression, produces mainly IMEs there. A more subtle difference is the slightly more complete burning in the He detonation of the ELDD-S model (compared to the other two models). This is a consequence of an additional compression of the hot He detonation ashes due to an oblique shock wave, which is sent from the core detonation into the shell.

\subsection{Synthetic observables}

The synthetic light curves of our models roughly match those of observed thermonuclear transients (see Fig. 2). The aforementioned differences in the final ejecta structure and composition have important consequences for the observable predictions of our models and might allow us to distinguish the different explosion scenarios observationally.

The light curves of the edge-lit model are very similar to those of the pure He detonation. Both models are compatible with previous results for the point-Ia scenario [3]. The presence of IMEs in 


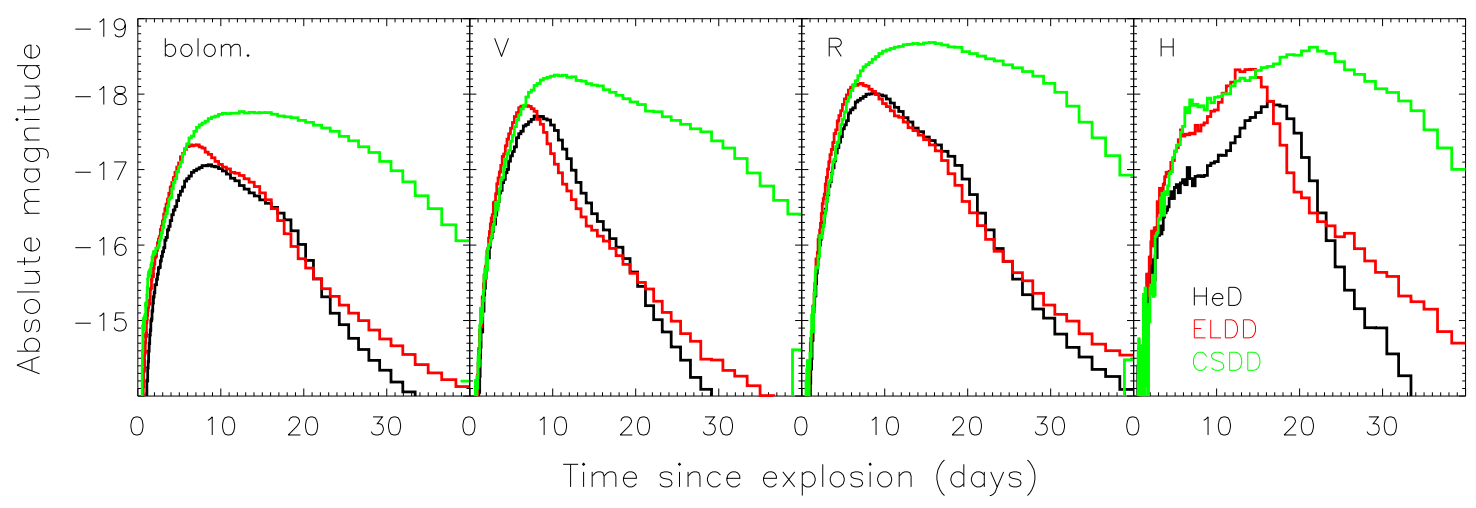

Figure 2: Angle-averaged synthetic light curves for the S models.

the core of model ELDD-S does not have a big influence on the optical light curves. However, in the near-infrared, it may lead to observable differences (see [17] for details).

Compared to the other two models, the CSDD-S model reaches significantly higher maximum brightnesses and the light curve evolves more slowly. This is due to the additional energy release of the ${ }^{56} \mathrm{Ni}$ produced in the core detonation. This energy takes a while to diffuse out.

\section{Summary and remarks on nucleosynthesis}

Sub-Chandrasekhar double detonations have been shown to be a robust explosion scenario that could explain the observed brightness range of Type Ia supernovae (provided that He shell detonations occur). Model changes that lead to less complete burning in the He shell detonations may improve the agreement with observations. From a nucleosynthesis point of view, sub-Chandrasekhar models have the advantage that their low-density $\mathrm{CO}$ detonations produce a lower ratio of ${ }^{58} \mathrm{Ni}$ to ${ }^{56} \mathrm{Ni}$ than $\mathrm{M}_{\mathrm{Ch}}$ models (see e.g. [24]), which is more consistent with Galactic chemical evolution (see e.g. [25]).

Low-mass double-detonation sub-Chandrasekhar models have been shown to produce light curves that resemble those of faint thermonuclear transients. The ELDD scenario is an interesting candidate for point-Ia like transients, whereas the CSDD scenario could produce brighter, more slowly evolving events. Finally, as the progenitors of these models may be very frequent, one might speculate that they could be important sources for the production of ${ }^{52} \mathrm{Cr}$ and ${ }^{48} \mathrm{Ti}$ (from ${ }^{52} \mathrm{Fe}$ and ${ }^{48} \mathrm{Cr}$ decay).

\section{Questions from the audience}

A. Heger: "What evolution scenario gives you a $0.45 \mathrm{M}_{\odot} \mathrm{CO}$ white dwarf?"

Answer: As commented by S. Sim, this CO-core mass is at the lower limit predicted by population synthesis studies of A. Ruiter [4]. It is reached in systems with $\mathrm{He}$ star or $\mathrm{He}-\mathrm{CO}$ hybrid WD donors that have low initial semi-latera recta $l$ and undergo only a single common 
envelope event. ${ }^{1}$ A low value of $l$ means either a low initial separation in general or at least a low minimum separation in a system with high eccentricity.

F. Thielemann: "In the first part of your talk you showed nicely that a reduced size of the He-shell (at ignition) can avoid the overproduction of $\mathrm{Ni}$ in the outer ejecta. What is the main reason to have an ignition possible for such smaller He-shells in comparison to earlier models?"

Answer: Our study [5] assumes setups with minimum He-shell mass as calculated by Bildsten et al. [2]. It is not well explored if these minimum He-shell masses are realised in nature, but it makes sense to study these systems as limiting case in He-explosion strength. Recently, lower mass He shells than in previous studies were also found by Woosley \& Kasen [26]. Compared to earlier results [9], they had used finer zoning during the accretion, included an additional reaction sequence ${ }^{12} \mathrm{C}(p, \gamma){ }^{13} \mathrm{~N}(\alpha, p){ }^{16} \mathrm{O}$, which facilitates He detonation initiation, and considered hotter (younger) CO-WD cores, which leads to a lower heat flow from the shell into the core.

\section{Acknowledgments}

This work was supported by the Emmy Noether Program (RO 3676/1-1). MF thanks the organisers of NIC and the DAAD for financial support.

\section{References}

[1] W. Li, J. Leaman, R. Chornock, A. V. Filippenko, D. Poznanski et al. Nearby supernova rates from the Lick Observatory Supernova Search - II. The observed luminosity functions and fractions of supernovae in a complete sample. MNRAS, 412 (2011) 1441. [1 006 . 4612] .

[2] L. Bildsten, K. J. Shen, N. N. Weinberg and G. Nelemans. Faint Thermonuclear Supernovae from AM Canum Venaticorum Binaries. ApJ, 662 (2007) L95. [arXiv: astro-ph/ 0703578 ] .

[3] K. J. Shen, D. Kasen, N. N. Weinberg, L. Bildsten and E. Scannapieco. Thermonuclear .Ia Supernovae from Helium Shell Detonations: Explosion Models and Observables. ApJ, 715 (2010) 767. [1002.2258].

[4] A. J. Ruiter, K. Belczynski, S. A. Sim, W. Hillebrandt, C. L. Fryer et al. Delay times and rates for Type Ia supernovae and thermonuclear explosions from double-detonation sub-Chandrasekhar mass models. MNRAS, (2011) 1282. [1011.1407].

[5] M. Fink, F. K. Röpke, W. Hillebrandt, I. R. Seitenzahl, S. A. Sim et al. Double-detonation sub-Chandrasekhar supernovae: can minimum helium shell masses detonate the core? A\&A, $\mathbf{5 1 4}$ (2010) A53. [1002.2173].

[6] S. A. Sim, F. K. Röpke, W. Hillebrandt, M. Kromer, R. Pakmor et al. Detonations in Sub-Chandrasekhar-mass $C+O$ White Dwarfs. ApJ, 714 (2010) L52. [1003.2917] .

[7] P. Höflich and A. Khokhlov. Explosion Models for Type Ia Supernovae: A Comparison with Observed Light Curves, Distances, $H_{0}$, and $q_{0}$. ApJ, 457 (1996) 500. [arXiv: astro-ph/9602025] .

[8] P. Nugent, E. Baron, D. Branch, A. Fisher and P. H. Hauschildt. Synthetic Spectra of Hydrodynamic Models of Type IA Supernovae. ApJ, 485 (1997) 812. [arXiv: astro-ph/9612044] .

\footnotetext{
${ }^{1}$ In an ellipse with semi-major axis $a$ and eccentricity $e$ the semi-latus rectum is $l=a\left(1-e^{2}\right)$.
} 
[9] S. E. Woosley and T. A. Weaver. Sub-Chandrasekhar mass models for Type Ia supernovae. ApJ, 423 (1994) 371.

[10] E. Livne and D. Arnett. Explosions of Sub-Chandrasekhar Mass White Dwarfs in Two Dimensions. ApJ, 452 (1995) 62.

[11] M. Kromer, S. A. Sim, M. Fink, F. K. Röpke, I. R. Seitenzahl et al. Double-detonation Sub-Chandrasekhar Supernovae: Synthetic Observables for Minimum Helium Shell Mass Models. ApJ, 719 (2010) 1067. [1006.4489].

[12] J. Guillochon, M. Dan, E. Ramirez-Ruiz and S. Rosswog. Surface Detonations in Double Degenerate Binary Systems Triggered by Accretion Stream Instabilities. ApJ, 709 (2010) L64. [0911. 0416 ] .

[13] R. Pakmor, M. Kromer, S. Taubenberger, S. A. Sim, F. K. Röpke et al. Normal Type Ia Supernovae from Violent Mergers of White Dwarf Binaries. ApJ, 747 (2012) L10. [1201 . 5123] .

[14] G. J. Sharpe. The effect of curvature on detonation waves in Type Ia supernovae. MNRAS, 322 (2001) 614.

[15] H. B. Perets, A. Gal-Yam, P. A. Mazzali, D. Arnett, D. Kagan et al. A faint type of supernova from a white dwarf with a helium-rich companion. Nature, 465 (2010) 322. [0906. 2003 ] .

[16] M. M. Kasliwal, S. R. Kulkarni, A. Gal-Yam, P. E. Nugent, M. Sullivan et al. Calcium-rich Gap Transients in the Remote Outskirts of Galaxies. ApJ, 755 (2012) 161. [1111.6109].

[17] S. A. Sim, M. Fink, M. Kromer, F. K. Röpke, A. J. Ruiter et al. 2D simulations of the double-detonation model for thermonuclear transients from low-mass carbon-oxygen white dwarfs. MNRAS, 420 (2012) 3003. [1111.2117].

[18] M. Reinecke, W. Hillebrandt and J. C. Niemeyer. Refined numerical models for multidimensional type Ia supernova simulations. A\&A, 386 (2002) 936. [arXiv: astro-ph/0111475].

[19] F. K. Röpke. Following multi-dimensional type Ia supernova explosion models to homologous expansion. A\&A, 432 (2005) 969. [arXiv:astro-ph/0408296].

[20] I. R. Seitenzahl, F. K. Röpke, M. Fink and R. Pakmor. Nucleosynthesis in thermonuclear supernovae with tracers: convergence and variable mass particles. MNRAS, 407 (2010) 2297. [1 005.5071 ] .

[21] S. A. Sim. Multidimensional simulations of radiative transfer in Type Ia supernovae. MNRAS, 375 (2007) 154. [arXiv:astro-ph/0611677].

[22] M. Kromer and S. A. Sim. Time-dependent three-dimensional spectrum synthesis for Type Ia supernovae. MNRAS, 398 (2009) 1809. [0906.3152] .

[23] I. R. Seitenzahl, C. A. Meakin, D. M. Townsley, D. Q. Lamb and J. W. Truran. Spontaneous Initiation of Detonations in White Dwarf Environments: Determination of Critical Sizes. ApJ, 696 (2009) 515. [0901.3677].

[24] I. R. Seitenzahl, F. Ciaraldi-Schoolmann, F. K. Roepke, M. Fink, W. Hillebrandt et al. Three-dimensional delayed-detonation models with nucleosynthesis for Type Ia supernovae. ArXiv e-prints, (2012). [1211.3015].

[25] C. Kobayashi, H. Umeda, K. Nomoto, N. Tominaga and T. Ohkubo. Galactic Chemical Evolution: Carbon through Zinc. ApJ, 653 (2006) 1145. [arXiv: astro-ph/ 0608688 ] .

[26] S. E. Woosley and D. Kasen. Sub-Chandrasekhar Mass Models for Supernovae. ApJ, 734 (2011) 38. [1010.5292]. 